Chirurg 2021 - 92:962

https://doi.org/10.1007/s00104-021-01488-5

Angenommen: 19. Juli 2021

Online publiziert: 11. August 2021

(c) Springer Medizin Verlag $\mathrm{GmbH}$, ein Teil von Springer Nature 2021

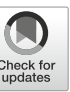

Check tor
updates

\section{Anastomosentechnik der Ösophagektomie mit gastraler Rekonstruktion - zervikal oder intrathorakal?}

\author{
W. Schröder · C. J. Bruns
}

Klinik für Allgemein-, Viszeral- und Tumorchirurgie, Universitätsklinik Köln, Köln, Deutschland
Originalpublikation

Van Workum F, Verstegen HP, Bastian R et al (2021) Intrathoracic vs cervical anastomosis after totally or hybrid minimally invasive esophagectomy for esophageal cancer. A randomized clinical trial. JAMA Surg. https:// doi.org/10.1001/jamasurg.2021.1555

Hintergrund und Fragestellung. Für die gastrale Rekonstruktion nach transthorakaler Ösophagektomie stehen grundsätzlich zwei verschiedene Möglichkeiten zur Verfügung, die intrathorakale (IvorLewis-) und zervikale (McKeown-)Ösophagogastrostomie. In den letzten Jahren hat sich in den Zentren für das mittlere und distale Ösophaguskarzinom zunehmend die intrathorakale Rekonstruktion etabliert, ohne dass für dieses Vorgehen hinreichend Evidenz besteht. Für die minimal-invasive Ösophagektomie wird zudem die intrathorakale Anastomose als technisch schwierig beurteilt und zeigt in Registeranalysen eine höhere Insuffizienzrate als beim offenen Vorgehen. In einer holländischen multizentrischen Studie wurden jetzt in einem prospektivrandomisierten Studiendesign die beiden Anastomosentechniken vergleichend untersucht.

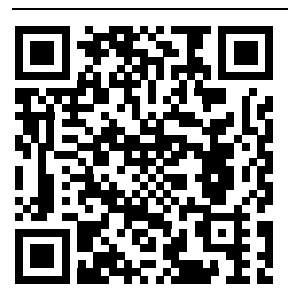

QR-Code scannen \& Beitrag online lesen
Methoden. In 9 holländischen High-volume-Zentren wurden über einen Zeitraum von 5 Jahren insgesamt 262 Patienten mit Ösophaguskarzinom randomisiert, $245 \mathrm{~Pa}$ tienten kamen nach Hybrid- oder total minimal-invasiver Ösophagektomie zur Auswertung. Von diesen wurden 122 Patienten intrathorakal und 123 zervikal rekonstruiert. Primärer Endpunkt war die Anastomoseninsuffizienz (Al), die endoskopisch oder chirurgisch therapiert wurde. Als sekundäre Endpunkte wurden die Rate anderer Komplikation, die Krankenhausmortalität, die Krankenhausaufenthaltsdauer und Lebensqualität 6 Wochen postoperativ (European Organization for Research and Treatment of Cancer - Quality of Life [EORTC-QLQ] C30 und OG25) dokumentiert und ausgewertet.

Ergebnisse. Als wesentliches Ergebnis dieser Studien konnte gezeigt werden, dass die Al-Rate mit notwendiger Intervention nach intrathorakaler Rekonstruktion signifikant geringer war (12,3\% vs. $31,7 \%$, $p<0,01)$. Auch die Rate der schweren Komplikationen (Clavien-Dindo $\geq 3 \mathrm{~b}$ ) war in der Ivor-Lewis-Kohorte signifikant niedriger $(10,7 \%$ vs. $22,0 \%, p=0,02)$. Krankenhaus- und Intensivaufenthalt waren in beiden Gruppen vergleichbar kurz, ebenso die 30- und 90-Tage-Mortalität vergleichbar niedrig (90-Tage-Mortalität: 3,3\% vs. $1,6 \%, p=0,4)$. Die chirurgisch-onkologischen Daten (resezierte Lymphknoten, R0Resektionsrate) waren für beide untersuchte Gruppen ebenfalls gleich. Wie zu erwarten, wurde nach zervikaler Anastomosierung bei 7,3\% der Patienten eine Parese des N. laryngeus recurrens diagnostiziert, in der Ivor-Lewis-Gruppe trat diese Komplikation nicht auf. Hinsicht der Lebensqualität waren die Gesamtscores im früh postoperativen Verlauf vergleichbar, allerdings zeigten sich in drei Subdomänen (Schluckbeschwerden, Sprechstörungen, Würgen) bessere Indizes für die intrathorakale Rekonstruktion.

\section{Kommentar}

In dieser einfach, aber gut konzipierten Studie wird wissenschaftlich untermauert, was in den letzten 10 Jahren in der Ösophaguschirurgie zunehmend in den spezialisierten Zentren praktiziert wird, nämlich die intrathorakale Rekonstruktion im oberen Mediastinum. Dies gilt für die minimal-invasive (thorakoskopische) und Hybrid-Ivor-Lewis-Ösophagektomie. Aufgrund dieser eindeutigen Daten ist für das mittlere und distale Ösophaguskarzinom dieses Verfahren als onkologischer Standard zu fordern und bei der nächsten Aktualisierung in die Leitlinie mit aufzunehmen.

\section{Korrespondenzadresse}

Prof. Dr. W. Schröder, FACS FEBS

Klinik für Allgemein-, Viszeral- und Tumorchirurgie, Universitätsklinik Köln Kerpener Str. 62, 50937 Köln, Deutschland wolfgang.schroeder@uni-koeln.de

Interessenkonflikt. W. Schröder und C. J. Bruns geben an, dass kein Interessenkonflikt besteht. 